\title{
A entrevista de crianças com suspeita de abuso sexual
}

\author{
Interviewing alleged victims \\ of child sexual abuse
}

Luis Roberto BENIA ${ }^{1}$

\section{Resumo}

O presente trabalho tem como objetivo revisar a literatura científica referente às melhores práticas na entrevista de investigação do abuso sexual infantil. Foi realizada uma revisão narrativa da literatura sobre as particularidades da memória e das habilidades de comunicação da criança em situação de entrevista investigativa, bem como as diferentes técnicas que permitem aprimorar a qualidade dos relatos infantis e maximizar a quantidade de informações obtidas. Foram pesquisadas as seguintes bases de dados: MedLine, PsychInfo e SciELO, no período de 1995 a 2012, utilizandose as palavras-chave "sexual abuse", "children", "interview", "investigative" e "forensic". Foram encontradas 196 referências, das quais 34 foram selecionadas para inclusão neste artigo. Foi encontrado grande número de evidências demonstrando a necessidade de procedimentos cuidadosos para avaliar as suspeitas de abuso sexual. Técnicas específicas são descritas, assim como são discutidas as dificuldades frequentemente encontradas pelos entrevistadores.

Palavras-chave: Abuso da criança; Abuso sexual; Entrevista.

\begin{abstract}
The aim of this study was to conduct a literature review to describe the best practice guidelines to evaluate sexual abuse interviews. A narrative literature review was carried out to establish the children's memory and communicative skills during an investigative interview, as well as the interview techniques that improve the quality of children's reports and maximize the amount of information elicited. We used the following electronic on-line databases: MedLine, Psychlnfo and ScIELO, referring to the period 1995-2012. The keywords searched for in the databases were "sexual abuse", "children", "interview", "investigative", "forensic". A total of 196 references were identified and 34 were selected for this paper. We found a large amount of evidence that recommends careful procedures when evaluating child sexual abuse. We describe the specific techniques and discuss the most common difficulties interviewers face during interviews.
\end{abstract}

Keywords: Child abuse; Sexual abuse; Interview.

Os crimes sexuais contra a criança são, por sua natureza, crimes silenciosos, sem testemunhas, o que impõe uma série de dificuldades para sua investigação. A denúncia, geralmente, ocorre após

\section{V V V}

1 Universidade Federal do Rio Grande do Sul, Instituto Geral de Perícias do Estado do Rio Grande do Sul, Departamento Médico-Legal. Av. Ipiranga, 1807, 90160-093, Porto Alegre, RS, Brasil. E-mail: <luis-benia@igp.rs.gov.br>. 
longo período de tempo (London, Bruck, \& Wright, 2008). Na maioria dos casos, não há evidências no exame físico, assim como o diagnóstico baseado nos sintomas psíquicos não diferencia de forma confiável as crianças abusadas daquelas que nunca sofreram abuso. Os achados psicológicos são na maioria das vezes inconclusivos, porque o abuso sexual pode causar uma grande variedade de quadros clínicos que também são encontrados em outras situações traumáticas na infância (Habigzang \& Caminha, 2004). Também não há um conjunto de alterações emocionais e comportamentais específicas do abuso sexual. A intensidade dos sintomas é variável, e algumas crianças podem não apresentar sintomas aparentes em um primeiro momento (Welter \& Feix, 2010). Constatada determinada sintomatologia na criança, ainda que possa sinalizar a associação a um possível abuso, ela não constitui evidência suficiente para informar acerca da ocorrência concreta de violência, tal qual se faz necessário para o deslinde de um processo judicial.

Portanto, na maioria dos casos, o relato da criança é a principal evidência pela qual poderá ser avaliada a validade de uma alegação de abuso sexual. As declarações da vítima se tornam, dessa forma, decisivas no desfecho de uma investigação criminal e de um processo judicial (Daltoé-Cezar, 2007; London et al., 2008;), como se pode observar neste acórdão do Tribunal de Justiça do Rio Grande do Sul:

Nos crimes sexuais, a palavra da vítima assume vital importância, sendo, muitas vezes, a única prova a determinar a condenação do réu. Pela sua natureza, tais infrações normalmente são cometidas de forma clandestina, longe dos olhos de qualquer testemunha. Assim, em regra, quando o relato da ofendida mostra-se firme e coerente, deve prevalecer no confronto com a versão defensiva (Piazzeta, 2012).

Nem sempre é fácil obter a declaração de uma criança vítima, porque ela tende a se sentir inibida diante de adultos que não conhece (Lamb, Sternberg, \& Esplin, 1998) e, muitas vezes, não está disposta a relatar experiências que são fonte 28 de ansiedade, vergonha ou culpa (Leander,
Christianson, \& Granhag, 2007). A criança necessita de um ambiente acolhedor e de profissionais preparados, que possam oferecer suporte emocional para que ela se sinta o mais confortável possível ao relatar experiências traumáticas.

Contudo, essa não é a realidade encontrada na maior parte das delegacias de polícia e das salas de audiência do País (Santos \& Gonçalves, 2008). As crianças com suspeita abuso sexual costumam ser escutadas como qualquer pessoa adulta que tenha se envolvido em uma situação ilícita (Froner $\&$ Ramires, 2008). Além do sofrimento emocional causado pela situação, pesquisas demonstram que esse tipo de inquirição é altamente improdutivo (Lamb, Hershkowitz, Orbach, \& Esplin, 2008), levando a resultados questionáveis do ponto de vista jurídico. Ou seja, dois aspectos devem ser atendidos: o cuidado para garantir a integridade psíquica da criança e, ao mesmo tempo, o cuidado para garantir a validade e a confiabilidade de seu relato. Se a palavra da vítima tem alto valor probatório, é preciso maximizar sua capacidade de narrar a experiência e, ao mesmo tempo, assegurar-se de que a informação fornecida corresponda a uma recordação realmente vivenciada. Em última instância, a obtenção de um relato de qualidade é crucial para a proteção da criança, pois reduz a impunidade dos agressores e, consequentemente, a perpetuação da violência.

A avaliação do abuso sexual infantil envolve diferentes profissionais: psiquiatras, psicólogos, assistentes sociais e pediatras que atuam em serviços de proteção à criança ou em serviços periciais e forenses. Além deles, há outros profissionais que, mesmo não exercendo uma atividade dirigida a tal fim, poderão ser eventualmente convocados a emitir parecer sobre um determinado caso.

Este artigo tem por objetivo revisar a literatura nacional e internacional relativa à entrevista com crianças com suspeita de abuso sexual. Pretende-se, dessa forma, contribuir para fomentar a discussão acerca do assunto entre os profissionais da área da saúde e, quiçá, acessar outros setores da extensa rede de agentes envolvidos em tais investigações, como o Conselho Tutelar, a Polícia, o Ministério Público e o Judiciário. 


\section{Método}

Trata-se de revisão narrativa da literatura, entendida como uma publicação de abordagem ampla, empregada para delinear e debater o desenvolvimento de um tema, sob determinada abordagem teórica ou contextual. Essa abordagem não possui metodologia que permita a reprodução quantitativa de respostas para questões específicas, porém permite a atualização de conhecimentos sobre determinando tema (Rother, 2007).

Realizou-se busca, em bancos de dados eletrônicos, por artigos publicados no período de 1995 a 2012. As palavras-chave utilizadas na revisão foram "sexual abuse", "children", "interview", "investigative" e "forensic". Foram pesquisadas as bases de dados eletrônicas MedLine-PubMed, SciELO e Psychinfo. Também foi realizada busca manual de publicações científicas, livros e textos referentes à temática em estudo.

Foram identificadas 196 publicações, incluindo artigos originais, revisões de literatura e livros. Uma análise inicial foi realizada com base nos títulos dos manuscritos e nos resumos daqueles que não permitiam ter certeza sobre sua inclusão neste estudo. Foram incluídas 34 publicações que se mostraram relevantes para o presente artigo. As referências selecionadas foram analisadas e organizadas em três eixos temáticos: as vulnerabilidades da criança quando está sendo entrevistada, a sugestionabilidade infantil e as técnicas de entrevista recomendadas pela literatura científica para reduzir a revitimização e melhorar a qualidade dos relatos infantis.

\section{Vulnerabilidades da criança em situação de entrevista}

O momento da entrevista com uma criança vítima de violência sexual pode ser entendido como um processo de recuperação da memória para o evento em questão. A literatura sugere que crianças, até as muito jovens, podem recordar e relatar detalhes importantes de eventos (Gordon, Baker-Ward, \& Ornstein, 2001; Lamb et al., 2008). No entanto, são necessários procedimentos investi- gativos cuidadosos para evitar os vários problemas que prejudicam a confiabilidade do testemunho infantil. O uso de técnicas inadequadas para a coleta das informações contidas na memória resultará em problemas na qualidade do relato e, consequentemente, diminuição da sua credibilidade e valor probatório. Muitos profissionais de saúde com experiência no atendimento a crianças têm dificuldade em conduzir uma entrevista quando o objetivo principal é a obtenção de informações acuradas sobre a ocorrência de um crime sexual (Orbach et al., 2000).

Na linguagem do dia a dia, o adulto modifica seu padrão discursivo quando conversa com uma criança. Tais características são facilmente observáveis nas falas de pais, professores e adultos em geral. Nesse tipo de conversação, a criança está habituada a ser "testada" pelo adulto (e.g. "qual a cor daquele carro?"); logo, ela frequentemente pressupõe que o adulto já saiba a resposta e que o objetivo da conversação seja descobrir qual é a resposta "certa", ou seja, qual a resposta que o adulto está esperando (Bruck \& Ceci, 1995; Lamb et al., 1998). A forma como um entrevistador coloca suas perguntas pode, inadvertidamente, levar a criança a responder com se estivesse sendo testada. A repetição de perguntas, tão comum nas conversas com criança, pode levá-la a crer que sua primeira resposta não era correta e a modificá-la. Repetir perguntas em uma mesma entrevista dirige a atenção da criança para sua resposta anterior, e não para a memória do que realmente aconteceu (Piper, 2008). Muitas crianças relutam em dizer "eu não sei" para perguntas muito específicas, principalmente as que exigem respostas sim/não, e podem responder do modo como acreditam que irá satisfazer o entrevistador (Lamb, Malloy, \& La Rooy, 2011). A criança não está acostumada a ser considerada única fonte de informação sobre um determinado assunto (Lamb et al., 2011). Por isso, é importante que ela compreenda que o entrevistador não detém o conhecimento sobre que aconteceu; caso contrário, poderá não revelar tudo o que sabe e também ficará mais suscetível à influência dele.

Frequentemente, as crianças, principalmente pré-escolares, utilizam palavras sem entender completamente o seu significado ou cujo significado 
para elas é diferente daquele para o adulto. Muitas vezes, o adulto superestima a capacidade linguística da criança e utiliza palavras, conceitos ou estruturas frasais que excedem sua capacidade de compreensão. Na tentativa de demonstrar sua cooperação com o adulto, a criança dificilmente responde "não entendi", mesmo quando não compreende as perguntas (Lamb et al., 2011). Crianças pequenas podem até mesmo não perceber que não entenderam porções significativas da pergunta e tentar responder mesmo assim (Waterman, Blades, \& Spencer, 2000). Problemas na compreensão mútua podem levar a distorções nas respostas e, o que é pior, tais distorções podem ser incorporadas em relatos subsequentes da criança (Hunt \& Borgida, 2001).

Certos conceitos, como tempo e número, são particularmente difíceis para uma criança compreender e utilizar corretamente em uma conversação. A capacidade de localizar eventos no tempo se desenvolve ao longo dos anos e demanda conhecimento de padrões temporais (Friedman \& Lyon, 2005). Pré-escolares frequentemente se confundem com termos tais como "antes", "depois", "próximo", "primeiro" e "último". Também é problemático perguntar a uma criança quantas vezes um determinado incidente ocorreu, pois ela provavelmente dirá um número qualquer (Wynn, 1992).

Os adultos geralmente fazem comentários para recompensar respostas ou conduzem a interação com foco em seu interesse, limitando a produção narrativa da própria criança. O resultado desse reforço seletivo é que a criança responderá de modo consistente com as convicções do adulto, ou suas respostas simplesmente refletirão informações obtidas das perguntas dos adultos (Bruck \& Ceci, 1995). Em contextos forenses, foi observado que, muitas vezes, o entrevistador tem prévia convicção sobre a ocorrência de certo evento e, como resultado, molda a entrevista para produzir declarações do entrevistado consistentes com essa convicção (Bruck, Ceci, \& Hembrooke, 2002). Nesse caso, o entrevistador não faz perguntas que poderiam fornecer explicações alternativas, nem indaga sobre eventos incompatíveis com sua hipótese. Quando a criança fornece evidência incompatível 30 com sua convicção, ele a ignora ou a interpreta de acordo com sua hipótese inicial. Geralmente, esse tipo de influência do entrevistador não ocorre de forma consciente ou deliberada: ele não percebe que está conduzindo a entrevista de modo a se encaixar em suas hipóteses pré-formadas (Nickerson, 1998).

\section{A sugestionabilidade infantil}

Indivíduos de todas as idades, com o passar do tempo, fornecem menor quantidade de informação, devido ao enfraquecimento do traço de memória. Crianças tendem a esquecer mais rapidamente do que adultos. Quando se trata de eventos estressantes ou emocionalmente agradáveis, foi demonstrado que crianças, mesmo as muito jovens, podem recordar aspectos centrais do ocorrido por longos períodos de tempo (Gordon et al., 2001). A memória, com o tempo, torna-se mais reconstrutiva e menos reprodutiva à medida que se vão introduzindo novas experiências, de modo que, quanto mais tempo transcorre entre o momento do evento e o da recordação, maior será a probabilidade de distorções (Duarte \& Arboleda, 2000). A sugestionabilidade consiste na tendência de um indivíduo em incorporar informações distorcidas, provindas de fontes externas, às suas recordações pessoais (Welter \& Feix, 2010). Embora adultos e crianças sejam sugestionáveis, crianças são especialmente suscetíveis à introdução de informação falsa após um evento. O monitoramento da fonte, assim denominada, é a capacidade de um indivíduo determinar se uma dada recordação é decorrente de sua própria experiência ou de algo que Ihe foi dito, que ouviu falar ou que imaginou. Essa capacidade se desenvolve ao longo da infância, e os pré-escolares têm maior dificuldade para identificar a origem de suas lembranças, estando mais vulneráveis aos efeitos da sugestionabilidade (Bright-Paul, Jarrold, \& Wright, 2008).

A contaminação da memória pode ocorrer de várias maneiras, seja através de elementos inseridos nas próprias perguntas feitas à criança, seja nas expressões e comportamentos dos adultos. Quando um adulto se encontra em posição de autoridade - seja ele pai, mãe, professor, policial ou 
profissional de saúde -, a criança se sente compelida a aceitar sua construção implícita dos fatos (Pisa, 2006). Portanto, é de capital importância compreender que o modo como esses diferentes agentes abordam o tema do abuso com a criança, pode ter grande impacto em seus futuros relatos sobre o ocorrido.

A maneira pela qual uma criança pode ser sugestionada tem sido alvo de inúmeros estudos, e esgotar o tema foge ao escopo deste artigo. No que diz respeito ao contexto da entrevista, a repetição de perguntas, especialmente perguntas fechadas do tipo sim ou não, que incluem elementos não mencionados pela vítima, pode fazer com que a criança incorpore algum desses elementos em relatos futuros (Bruck \& Ceci, 1995; Duarte \& Arboleda, 2000; Lamb et al., 1998). A simples repetição de perguntas pode ter o efeito de sugerir para a criança que o entrevistador não está satisfeito com sua resposta inicial. Outra forma sutil de sugestão se dá quando o entrevistador confere uma atmosfera excessivamente emocional durante a entrevista. Presumindo estar estabelecendo uma relação encorajadora e suportiva, está, na realidade, configurando um tom sentimental à entrevista, pelo uso, implícito ou explícito, de ameaças, subornos e recompensas. Declarações como "eu sei que algo ruim aconteceu" ou "não tenha medo de contar tudo" podem expor as crenças do entrevistador, ao pressupor que algo de fato aconteceu (Pisa, 2006). Do mesmo modo, a indução de um estereótipo do suposto agressor pode influenciar as respostas da criança. Isso ocorre quando o entrevistador transmite uma ideia ou uma caracterização negativa do suspeito, com o intuito de encorajar a criança a revelar o abuso. Algumas crianças podem vir a interpretar falsamente um evento como sendo negativo, com base em estereótipos negativos posteriores ao evento, que são transferidos a elas por entrevistadores, inclusive familiares, professores ou outros técnicos (Bruck \& Ceci, 1995).

\section{Técnicas de entrevista baseadas em evidências}

Atualmente, há um consenso na literatura internacional em como uma entrevista investigativa deve ser conduzida. As melhores práticas baseadas em evidências são descritas a seguir.

- Ambientação: As diretrizes recomendam um ambiente com privacidade, que seja acolhedor para uma criança, mas com um mínimo de distrações. Estudos indicam que elementos que distraem a criança (brinquedos, desenhos, etc.) exigem que ela divida sua atenção, prejudicando sua capacidade de se concentrar na entrevista (Poole \& Lamb, 1998). A privacidade é essencial tanto para evitar distrações, quanto para evitar possíveis contaminações por parte dos adultos que acompanham a criança. Nos casos em que a criança esteja muito insegura e se recuse a ficar a sós com o entrevistador, autoriza-se a presença de um acompanhante, solicitando-lhe que se posicione atrás da criança, que permaneça em silêncio e que redirecione a atenção dela para o entrevistador quando necessário (Saywitz, Lyon, \& Goodman, 2010).

- Início da entrevista: O entrevistador deve utilizar os momentos iniciais da entrevista para estabelecer um bom vínculo com a criança e dar-lhe suporte emocional para que ela se sinta menos desconfortável com a situação. Uma postura de escuta empática auxilia na construção de uma relação calorosa e favorece a introdução de assuntos mais delicados. Ao mesmo tempo, o entrevistador deve tomar cuidado para manter sua neutralidade e não dar um tom sentimental à entrevista. Como visto anteriormente, o tom emocional conferido pelo entrevistador pode produzir reforço seletivo de respostas que se encaixem em suas crenças sobre os fatos.

Estudos têm demonstrado que um bom vínculo inicial facilita a comunicação entre a criança e o entrevistador, que produz relatos mais extensos e mais detalhados dos eventos, e que aumenta a acurácia das informações (Hershkowitz, Orbach, Lamb, Sternberg, \& Horowitz, 2006; Sternberg et al., 1997).

- Dar permissão explícita para a criança dizer "eu não sei" e "eu não entendi": As crianças estão acostumadas a interagir com adultos que já sabem as respostas para as perguntas que elaboram e, em razão disso, tendem a tentar adivinhar a resposta que imaginam que o adulto esteja esperando. Elas 
apresentam certa relutância em admitir ignorância ou incapacidade de recordar algo e não têm o hábito de pedir que o adulto esclareça uma pergunta que não entenderam. Elas simplesmente respondem da forma que supõem ser a mais correta. Vários estudos indicam que instruir a criança sobre a possibilidade de responder que não sabe ou que não entendeu a questão aumenta a acurácia dos relatos (Saywitz et al., 2010).

É importante que o entrevistador diga para a criança que ele não sabe o que aconteceu. Informar à criança que somente ela detém o conhecimento dos fatos melhora a qualidade dos relatos infantis (Lamb et al., 1998).

- Prática narrativa: As crianças, principalmente em idade pré-escolar, aportam menos quantidade de informação, pois não estão acostumadas a fornecer narrativas elaboradas sobre suas experiências. $O$ entrevistador deve mostrar à criança o quanto de detalhamento é esperado dela, deve exercitá-la a fornecer respostas descritivas e a elaborar narrativas sobre os eventos vividos com suas próprias palavras, usando perguntas abertas e não sugestivas. Esse tipo de exercício aumenta a quantidade de informação recuperada sobre o episódio de abuso sofrido (Sternberg et al., 1997) A prática narrativa consiste em solicitar à criança, no início da entrevista, que descreva eventos recentes (uma festa de aniversário, por exemplo) sob a forma de um relato livre e, na sequência, pedir a ela que forneça mais detalhes sobre o que aconteceu nesse evento. Então, a partir somente de detalhes que a criança descreveu, pedir a ela que fale mais a respeito deles, sempre utilizando perguntas abertas (Hershkowitz, 2011).

- Tipo de perguntas: É importante fazer uma distinção entre perguntas que testam a recordação livre e aquelas que testam a memória de reconhecimento. A recordação livre é acessada em condições nas quais não há qualquer indicação ou pista. O entrevistador pergunta algo como "o que aconteceu?" e deixa que criança forneça todos os detalhes. Toda a entrevista deve ser conduzida com base principalmente em perguntas abertas, gerais ou exploratórias, pois são essas que demandam a 32 recordação livre da criança.
Em contraste, a memória de reconhecimento é testada quando são oferecidas ao entrevistado duas ou mais opções de resposta que ele deve escolher. Perguntas fechadas, sugestivas, diretas ou de escolha forçada são perguntas de reconhecimento. As perguntas de reconhecimento incluem aquelas que são respondidas com $\operatorname{sim} /$ não e perguntas de escolha forçada ("era A ou B?"), cujas opções de resposta são fornecidas pelo entrevistador, e a criança apenas se limita a escolher entre as opções oferecidas (Pisa, 2006). Entrevistas baseadas nesse tipo de perguntas produzem relatos com menor precisão, menor quantidade de detalhes e têm grande potencial para sugestionar a criança, quando introduz elementos não referidos pela vítima (Lamb et al., 2008). Portanto, as perguntas fechadas, quando necessárias, devem ser feitas somente depois que a criança teve oportunidade para descrever os eventos com suas próprias palavras, a partir de perguntas abertas.

Quando a criança apresenta dificuldade em responder a perguntas abertas mais genéricas, recomenda-se que o entrevistador se refira aos elementos narrativos (ações, pessoas, lugares, objetos) que a criança mencionou e solicite a ela que elabore um pouco mais (ex.: fale mais sobre [elemento do relato da criança]). Para solicitar à criança uma narrativa mais extensa, os entrevistadores podem utilizar livremente a pergunta "o que aconteceu depois de [detalhe mencionado pela criança]?". Essa técnica melhora a capacidade da criança de reconstruir os eventos passados, ao estruturar a lembrança das experiências vividas, associando-as aos detalhes que ela mesma referiu (Orbach \& Pipe, 2011).

Outro tipo de pergunta que pode ser utilizada são aquelas que enfocam a atenção da criança em um tipo particular de resposta, permitindo-lhe preencher os detalhes. São perguntas do tipo "Wh" (do inglês what, where, when, who, why, ou how), ou seja, o que, onde, quando, que, por que, ou como (Pisa, 2006). Há certo risco com o uso desse tipo de questão em um formato muito específico, tal como "Qual a cor da roupa do homem?". Crianças com tendência a "adivinhar" podem fornecer respostas plausíveis, mas inventadas, apenas para satisfazer o entrevistador (Saywitz et al., 2010). 
Ainda assim, perguntas tipo "Wh" são consideradas menos sugestivas do que perguntas fechadas.

Em resumo, os modos de questionar uma criança se estendem ao longo de um contínuo em que, num extremo, a criança fornece todos os detalhes e, no outro, o entrevistador os fornece. Se a criança é solicitada a fornecer todos os detalhes, as perguntas são abertas. Quando as perguntas se posicionam na direção fornecida pelo entrevistador, elas se tornam mais fechadas e sugestivas.

- Estrutura da entrevista: Os diferentes modelos de entrevista variam desde as menos estruturadas, nas quais o entrevistador conduz a conversação sem uma ordem pré-estabelecida, até as completamente estruturadas, nas quais há uma sequência de etapas a serem seguidas e indicações de como os eventos devem ser abordados. Alguns estudos demonstraram que os entrevistadores têm dificuldade em aderir às praticas recomendadas quando estão em campo, a não ser que sigam uma abordagem estruturada ou, pelo menos, semiestruturada (Davies, Westcott, \& Horan, 2000; Sternberg, Lamb, Esplin, Orbach, \& Hershkowitz, 2002).

Dois modelos de entrevista largamente utilizados são a Entrevista Cognitiva (Memon, Meissner, \& Fraser, 2010) e o Protocolo de Entrevista Investigativa do National Institute of Child Health and Human Development (NICHD) (Lamb, Orbach, Hershkowitz, Esplin, \& Horowitz, 2007). Ambos propõem uma abordagem em etapas: a) fase preparatória, na qual o entrevistador se empenha em criar um vínculo com a criança, dá as instruções e propõe alguma prática narrativa; b) fase de relato livre, na qual o entrevistador solicita à criança que conte tudo o que aconteceu, sem interrompê-la; c) etapa de questionamento, para explorar os elementos narrados pela criança, mediante perguntas abertas e não sugestivas (perguntas fechadas, caso necessário, são feitas somente após essa etapa); d) fase de encerramento.

- Gravação da entrevista: Gravar a entrevista realizada com a criança traz uma série de vantagens importantes para a avaliação do abuso sexual. A existência de um depoimento gravado pode reduzir, em tese, o número de vezes que uma vítima é submetida a estressantes inquirições sobre um acontecimento traumático. Quando há uma entrevista de qualidade, diferentes agentes (Polícia, Ministério Público e Judiciário) poderão se valer do mesmo registro para avaliar o caso. A redução do número de entrevistas, além de reduzir o sofrimento da criança, diminui a possibilidade de que seu relato seja contaminado por influências externas. A gravação também encoraja o entrevistador a utilizar as técnicas mais adequadas ao documentar que ele conduziu a entrevista sem utilizar recursos sugestivos ou coercitivos para obter as informações (Poole \& Lamb, 1998). Gravar a fala da criança preserva a espontaneidade de seu relato, o modo como contextualiza os fatos, a quantidade de detalhes referidos e uma série de elementos narrativos que não podem ser reproduzidos em um relatório escrito durante ou após a entrevista.

\section{Considerações Finais}

A maior publicidade e a maior importância dada ao tema do abuso sexual provocou um aumento nas denúncias desse tipo de crime nos últimos anos. Com esse aumento, observa-se também um incremento no número de falsas denúncias. Os profissionais da saúde, da polícia e do judiciário se deparam, então, com a difícil tarefa de identificar os casos reais, tanto para proteger adequadamente as vítimas, quanto para evitar a punição de pessoas inocentes. Quando se trata de uma denúncia envolvendo pais ou pessoas da família, são particularmente desastrosas as consequências de se tomar como verdadeira uma denúncia falsa.

Para dar o devido crédito à palavra da vítima é preciso saber como conversar com ela sobre esse assunto tão difícil de abordar, a fim de obter as informações relevantes para comprovar a ocorrência do abuso sexual. No contexto brasileiro, com exceção de alguns poucos serviços, a maioria das crianças têm sido abordadas de forma imprópria, com perguntas incompreensíveis, inadequadas e intrusivas, muitas vezes de forma repetitiva, o que termina por causar maior sofrimento e comprometer a qualidade dos relatos. Uma entrevista bem conduzida, ao contrário, além de reduzir o desconforto da criança, dá consistência e confiabilidade a sua 
história. Entrevistar crianças, com essa perspectiva, demanda dos profissionais envolvidos conhecimentos específicos sobre a memória e as habilidades de comunicação infantis, bem como treinamento em técnicas de entrevista.

\section{Referências}

Bruck, M., \& Ceci, S. J. (1995). Jeopardy in the courtroom: A scientific analysis of children's testimony. Washington, DC: American Psychological Association.

Bruck, M., Ceci, S. J., \& Hembrooke, H. (2002). The nature of children's true and false narratives. Developmental Review, 22(3), 520-554.

Bright-Paul, A., Jarrold, C., \& Wright, D. B. (2008). Theoryof-mind development influences suggestibility and source monitoring. Developmental Psychology, 44(4), 1055-1068.

Daltoé-Cezar, J. A. (2007). Depoimento sem dano: uma alternativa para inquirir crianças e adolescentes nos processos judiciais. Porto Alegre: Livraria do Advogado.

Davies, G. M., Westcott, H. L., \& Horan, N. (2000). The impact of questioning style on the content of investigative interviews with suspected child sexual abuse victims. Psychology, Crime \& Law, 6(2), 81-97.

Duarte, J.C., \& Arboleda, M.R.C (2000). Guia para la evaluación del abuso sexual infantil. Madri: Pirámide.

Friedman, W. J., \& Lyon, T. D. (2005). Development of temporal reconstructive abilities. Child Development, 76(6), 1202-1216.

Froner, J. P., \& Ramires, V. R. (2008). Escuta de crianças vítimas de abuso sexual no âmbito jurídico:uma revisão crítica da literatura. Paidéia, 18(40), 267-278. Recuperado em agosto 10, 2012. de http://www.scielo.br/ pdf/paideia/v18n40/05.pdf

Gordon, B. N., Baker-Ward, L., \& Ornstein, P. A. (2001). Children's testimony: A review of research on memory for past experiences. Clinical Child and Family Psychology Review, 4(2), 157-181.

Habigzang, L. F., \& Caminha, R. M. (2004). Abuso sexual contra crianças e adolescentes: conceituação e intervenção clínica. São Paulo: Casa do Psicólogo.

Hershkowitz, I., Orbach, Y., Lamb, M. E., Sternberg, K. J., \& Horowitz, D. (2006). Dynamics of forensic interviews with suspected abuse victims who do not disclose abuse. Child Abuse \& Neglect, 30(7), 753-769.

Hershkowitz, I. (2011). Rapport Building in Investigative interviews of children. In M. E. Lamb, D. J. La Rooy, L. C. Malloy, \& C. Catz. (Orgs.), Children's testimony: A handbook of psychological research and forensic practice. Sussex: Wiley-Blackwell.
Hunt, J.S., \& Borgida, E. (2001). Is that what I said? Witnesses'responses to interviewer modifications. Law and Human Behavior, 25(6), 583-603.

Lamb, M. E., Malloy, L. C., \& La Rooy, D. J. (2011) Setting realistic expectations: Developmental characteristics, capacities and limitations. In M. E. Lamb, D. J. La Rooy, L. C. Malloy, L. C., \& C. Catz. (Orgs.), Children's testimony: A handbook of psychological research and forensic practice. Sussex: Wiley-Blackwell.

Lamb, M. E., Sternberg K. J., \& Esplin, P. W. (1998). Conducting investigative interviews of alleged sexual abuse victims. Child Abuse \& Neglect, 22(8), 813-823.

Lamb, M. E, Hershkowitz, I., Orbach,Y., \& Esplin, P. W. (2008). Tell me what happened: Structured investigative interviews of child victims and witnesses. West Sussex: Wiley.

Lamb, M., E., Orbach, Y., Hershkowitz, I., Esplin, P. W., \& Horowitz, D. (2007). Structured forensic interview protocols improve the quality and informativeness of investigative interviews with children: A review of the research using the NICHD Investigative Interview Protocol. Child Abuse and Neglect, 31(11-12), 1201-1231.

Leander, L., Christianson, S. A., \& Granhag, P. A. (2007). A sexual abuse case study: Children's memories and reports. Psychiatry, Psychology, and Law, 14(1), 120-129.

London, K., Bruck, M., \& Wright, D. B. (2008). Review of the contemporary literature on how children report sexual abuse to others: Findings, methodological issues, and implications for forensic interviewers. Memory, 16(1), 29-47.

Memon, A., Meissner, C.A., \& Fraser, J. (2010). The Cognitive Interview: A meta-analytic review and study space analysis of the past 25 years. Psychology, Public Policy, and Law, 16(4), 340-372.

Nickerson, R. S. (1998). Confirmation bias: A ubiquitous phenomenon in many guises. Review of General Psychology, 2(2), 175-220.

Orbach, Y., \& Pipe, M. E. (2011). Investigating substantive issues. In M. E. Lamb, D. J. La Rooy, L. C. Malloy, \& C. Catz (Orgs.), Children's testimony: A handbook of psychological research and forensic practice. West Sussex: Wiley-Blackwell.

Orbach, Y., Hershkowitz, I., Lamb, M. E., Sternberg, K. J., Esplin, P. W., \& Horowitz, D. (2000). Assessing the value of structured protocols for forensic interviews of alleged child abuse victims. Child Abuse \& Neglect, 24(6), 733-752.

Piazzeta, N. O. (2012). Apelação crime n 70046325700 , Tribunal de Justiça do Rio Grande do Sul - Sétima Câmara Criminal. Recuperado em 2012, agosto 1, de http://www.tjrs.jus.br/site/jurisprudencia

Piper, A. (2008). Investigating child sex abuse allegations: A guide to help legal professionals distinguish valid from invalid claims. The Journal of Psychiatry \& Law, 36(2), 271-317. 
Pisa, O. (2006). Psicologia do testemunho: os riscos na inquirição de crianças (Dissertação de mestrado não-publicada). Pontifícia Universidade Católica do Rio Grande do Sul, Porto Alegre.

Poole, D. A., \& Lamb, M. E. (1998). Investigative interviews of children: A guide for helping professionals. Washington, DC: American Psychological Association.

Rother, E. T. (2007). Revisão sistemática X revisão narrativa. Acta Paulista de Enfermagem, 20(2), 01-02.

Saywitz, K. J., Lyon, T. D., \& Goodman, G. S. (2010). Interviewing children. In J. E. B. Myers (Org.), The APSAC handbook on child maltreatment (pp.337-360). Thousand Oaks: Sage Publications.

Santos, B. R., \& Gonçalves, I. B. (2008). Depoimento sem medo? Culturas e práticas não-revitimizantes. São Paulo: Childhood Brasil.

Sternberg, K. J., Lamb, M. E., Hershkowitz, I., Yudilevitch L., Orbach, Y., Esplin, P. W., \& Hovav, M. (1997). Effects of introductory style on children's abilities to describe experiences of sexual abuse. Child Abuse \& Neglect, 21(2), 1133-1146.
Sternberg, K. J., Lamb, M. E., Esplin, P. W., Orbach, Y., \& Hershkowitz, I. (2002). Using a structured interview protocol to improve the quality of investigative interviews. In M. Eisen (Ed.), Memory and suggestibility in the forensic interview (pp.409-436). Mahwah, N.J: Lawrence Erlbaum Associates.

Waterman, A. H., Blades, M., \& Spencer, C. P. (2000). Do children try to answer nonsensical questions? British Journal of Developmental Psychology, 18(2), 211-226.

Welter, C. L. W, \& Feix, L. F. (2010). Falsas memórias, sugestionabilidade e testemunho infantil. In L. M. Stein (Org.), Falsas memórias: fundamentos científicos e suas aplicações clínicas e jurídicas (pp.157-185). Porto Alegre: Artmed.

Wynn, K. (1992). Children's acquisition of the number words and the counting system. Cognitive Psychology, 24(2), 220-251.

Recebido: novembro 14, 2012

Versão final: março 26, 2014

Aprovado: junho 9, 2014 
